\title{
ANIMALE, “TRANSANIMALE” E UMANO NEL PENSIERO DI HANS JONAS
}

\author{
Animal, "transanimal" e humano no pensamento de Hans Jonas
}

Roberto Franzini Tibaldeo

Università degli Studi di Torino

\begin{abstract}
II pensiero di Hans Jonas, specie per quel che riguarda la cosiddetta "biologia filosofica", tratta indirettamente del rapporto tra essere umano e animale. A questo riguardo, Jonas rifiuta sia l'approccio dualistico, sia quello monistico-riduzionistico e propende al contrario per una complessiva reinterpretazione del fenomeno della vita nei termini di quel che egli definisce una "rivoluzione ontologica". In virtù di ciò, il pensatore rintraccia lo specifico del fenomeno della vita e individua nelle forme viventi una scala naturae di complessità, auto-trascendimento e libertà via crescenti, le cui tappe significative sono la vita organica, quella animale e quella umana. Per quel che concerne la forma animale, varie specie presentano "potenzialità trans-animali", che evidenziano un ponte biologico e ontologico verso l'essere umano. In altre parole, l'animale è in qualche modo in grado di prefigurare la forma di vita specificamente umana. Tuttavia, sostiene Jonas, non appena quest'ultima fa la propria comparsa, essa è tale per cui se ne evidenzia al tempo stesso anche lo "iato metafisico" rispetto alla vita animale. La specificità umana si manifesta nella propria capacità di essere responsabile e di preservare le condizioni basilari per una vita autentica sul pianeta.
\end{abstract}

Keywords: Animale, transanimale, umano, Hans Jonas

Sumário: O pensamento de Hans Jonas, considerado a partir da assim chamada "biologia filosófica", trata indiretamente da relação entre ser humano e anima. Sobre isso, Jonas recusa tanto a abordagem dualistas quanto a monista-reducionista, propondo, ao contrário, uma complexa reinterpretação do fenômeno da vida nos termos do que ele define como uma "revolução ontológica". Em virtude disso, o pensador traça o específico do fenômeno da vida e individua nas formas vivas uma scala naturae de complexidade, autotranscendência e liberdade por uma via crescente, cuja nota significativa é a vida orgânica, animal e humana. No que concerne à forma animal, varias espécies apresentam "potencialidade trans-animal", que evidenciam uma ponte biológica e ontológica com o ser humano. Em outras palavras, o animal está de algum modo em condições de prefigurar a forma de vida especificamente humana. Todavia, sustenta Jonas, não apenas esta última segue a própria sequência, mas é tal que nela se evidencia ao mesmo tempo o "hiato metafísico" em relação à vida animal. A especificidade humana se manifesta na própria capacidade de ser responsável e de preservar as condições basilares para uma vida autêntica no planeta.

Palavras-Chaves: Animal, transanimal, humano, Hans Jonas 


\section{La "biologia filosofica" jonasiana: scala naturae e specifico animale}

Uno degli obiettivi della biologia filosofica di Jonas è di oltrepassare il dualismo cartesiano, e ciò sotto due aspetti: per un verso, l'autore mostra i limiti del dualismo psicofisico e, per altro verso, procede a una ridefinizione dello specifico dell'essere umano mettendolo in rapporto fecondo con il mondo naturale e vivente ${ }^{1}$.

Da questo punto di vista, Jonas - come molti esponenti dell'antropologia filosofica novecentesca ${ }^{2}$ - è restio a concedere alla tradizione cartesiana di aver pronunciato l'ultima parola riguardo all'essenza dell'umano e alla sua distinzione rispetto al mondo biologico, distinzione alla quale, peraltro, non si può rinunciare, pena lo smarrimento di ciò che lo caratterizza in quanto tale l'essere umano ${ }^{3}$. II punto decisivo è, pertanto, procedere a una rifondazione della specificità umana senza rimanere intrappolati nelle maglie del dualismo psicofisico e ontologico.

A questo riguardo, è inevitabile che il pensiero jonasiano si imbatta nell'evoluzionismo darwiniano, i cui risvolti filosofici - afferma Jonas - determinarono il definitivo tramonto della prospettiva dualistica cartesiana a mezzo però di una ridefinizione dell'umano in senso monistico, una ridefinizione che ricondusse l'essenza "duale" e "polare" di quest'ultimo a mera complicazione quantitativo-materiale ${ }^{4}$. Al di là delle puntuali critiche rivolte al darwinismo ${ }^{5}$, la biologia filosofica jonasiana analizza

\footnotetext{
${ }^{1}$ Cfr. H. Jonas, The Phenomenon of Life. Towards a Philosophical Biology, Harper \& Row, New York, 1966, ed. ted. con variazioni Organismus und Freiheit. Ansätze zu einer philosophischen Biologie, Vandenhoeck \& Ruprecht, Göttingen, 1973, poi riedita con il titolo Das Prinzip Leben. Ansätze zu einer philosophischen Biologie, Insel, Frankfurt am Main-Leipzig, 1994, tr. it. Organismo e libertà. Verso una biologia filosofica, a cura di P. Becchi, Einaudi, Torino, 1999.

2 Si vedano autori, quali Scheler, Plessner, Gehlen, Portmann. Cfr. J. Fischer, Philosophische Anthropologie. Eine Denkrichtung des 20. Jahrhunderts, Alber, Freiburg-München, 2008.

${ }^{3} \mathrm{H}$. Jonas, Organismo e libertà, cit., pp. 3-4.

${ }^{4}$ Sulla questione del monismo e sulla dinamica "polare" della vita, cfr. soprattutto ivi, pp. 95-129; sul darwinismo, cfr. ivi, pp. 52-74.

${ }^{5}$ Cfr. ivi, pp. 62 ss. Per le critiche avanzate da Jonas nei confronti del darwinismo, mi permetto di rinviare al mio La rivoluzione ontologica di Hans Jonas. Uno studio sulla genesi e il significato di "Organismo e libertà", Mimesis, Milano, 2009, pp. 243-255. Cfr. anche P. Becchi-R. Franzini Tibaldeo, Né darwinismo né Intelligent Design. Un confronto tra Hans Jonas e Joseph Ratzinger, in "Annuario filosofico", 29, 2015.
} 
attentamente le istanze evoluzionistiche, non ultima quella che postula la rilevanza dell'animale per la comprensione dell'umano. Nello specifico, l'attenta considerazione jonasiana dell'animale (in specie nelle sue manifestazioni "superiori") e delle sue potenzialità transanimali consente all'essere umano di fare luce sulla propria base biologica in quanto dotata di realtà e significato spirituale ${ }^{6}$.

Nell'ambito della biologia filosofica jonasiana l'animale si trova al centro di considerazioni riassumibili secondo due direzioni. Da un lato, infatti, esso presenta i medesimi tratti essenziali del vivente già prefigurati a livello metabolico-organico, quali l'interiorità e il plesso - caratteristico della vita in quanto tale - di polarità ontologica, dinamismo dialettico e ambivalenza interpretativa7. Dall'altro lato, però, il valore intrinseco manifestato dalla vita animale tende al di là di sé, a conferma di come, anche a questo stadio di sviluppo, la peculiare dinamica evidenziata dalla vita assuma la forma di una progressiva - e al tempo stesso sempre qualitativamente innovativa manifestazione del principio ontologico della libertà, di cui l'autore tenta di chiarire i contorni in Organismo e libertà (1966). Si tratta del carattere teleologico della vita, vale a dire del suo manifestarsi - all'osservatore - come una tendenza dinamica orientata, oltreché - relativamente a ciascun vivente - come un preoccuparsi della vita per se stessa e per le qualità via via conseguite ${ }^{8}$. Sotto questo profilo ciascuno stadio del vivente, così come ciascun essere vivente, evidenzia una tensione a trascendersi: nel singolo vivente, tale tensione trova concreta realizzazione in forme di relativa autonomia dalla propria base materiale ${ }^{9}$; al di là del singolo individuo, essa consente invece di distinguere diversi livelli di autonomia e di mostrare come essi possano

\footnotetext{
${ }^{6}$ Come si vedrà, questo non significa appiattire l'umano sulla propria base biologica, ma rimetterlo in contatto con quest'ultima. Nelle parole di P. Becchi: "Ma Jonas non accetta le implicazioni materialistiche e antiteleologiche del discorso di Darwin: non l'uomo viene ridotto al livello delle piante e degli animali, ma al contrario è l'intera natura vivente a essere elevata al livello umano. Non soltanto noi uomini siamo in possesso di interiorità, soggettività, libertà, ma in grado diverso tutti gli organismi viventi" (P. Becchi, Hans Jonas. Un profilo, Morcelliana, Brescia, 2010, p. 34).

${ }^{7} \mathrm{Cfr}$. H. Jonas, Organismo e libertà, cit., pp. 137-148.

${ }^{8}$ Cfr. ivi, p. 168.

${ }^{9}$ La struttura primordiale di tale relativa libertà è riscontrabile nell'organismo vivente, e precisamente nel rapporto sussistente tra forma e materia (cfr. ivi, pp. 106-116).
} 
venire ricompresi in una complessiva storia della vita in quanto avventura ontologica della libertà.

Relativamente a questo secondo aspetto, occorre ora interrogarsi sul senso attribuito al rapporto tra un livello e l'altro della progressione. Premesso che, per Jonas, nessun livello è riducibile a un prodotto causale-efficiente (ma neppure solo casuale) del precedente e posto che debba pur esserci tra i vari livelli un legame reale e intrinseco di qualche tipo, ecco che il Nostro pare risolvere la questione riportando in auge il concetto, di sapore aristotelico, di potenzialità ${ }^{10}$. Egli ne parla, tra I'altro, a proposito del legame dinamico-evolutivo sussistente tra animale e uomo: alcuni caratteri presentati dall'animale (nello specifico, il senso della vista) conterrebbero infatti "potenzialità transanimali di intuizione e attitudine, che una superiore facoltà spirituale può realizzare"11. E viceversa, la chiarificazione - nell'ambito di una

\footnotetext{
10 Alla trattazione della nozione aristotelica di "potenzialità" sono infatti dedicati alcuni passi di un inedito jonasiano risalente agli anni 1966-1967 conservato presso il Philosophisches Archiv dell'Università di Konstanz (Germania) e intitolato Life and Organism (cfr. il Nachlass jonasiano alla segnatura HJ-1-12-1, pp. $35 \mathrm{ss}$.). Jonas si vede in un certo senso costretto a ricorrere a questa ontologia "aristotelica" per lasciarsi alle spalle la sterile alternativa tra dualismo e monismo emergentista (à la Lloyd Morgan), che rischiava di travisare la rilevanza ontologica della teleologia presente in natura (cfr. H. Jonas, Das Prinzip Verantwortung. Versuch einer Ethik für die technologische Zivilisation, Insel, Frankfurt am Main, 1979, tr. it. Il principio responsabilità. Un'etica per la civiltà tecnologica, a cura di P. P. Portinaro, Einaudi, Torino, 1990, pp. 84-94). Non a caso Jonas qualifica la propria proposta come un "monismo integrale a un livello superiore" (H. Jonas, Organismo e libertà, cit., p. 27). E aggiunge: "Un nuovo monismo integrale, ossia filosofico, non può annullare la polarità, ma deve venirne a capo, deve superarla in una superiore unità dell'essere, dalla quale gli opposti escono come facce della sua realtà o fasi del suo divenire" (ivi, p. 25). Per una riflessione sul concetto di "emersione" in rapporto alla teoria dell'evoluzione e alla questione della libertà umana, cfr. B. Feltz, Self-Organization, Selection and Emergence in the Theories of Evolution, in B. Feltz-M. Crommelinck-Ph. Goujon (a cura di), SelfOrganisation and Emergence in Life Sciences, Springer, Dordrecht, 2006, pp. 341-360; B. Feltz, Plasticité neuronale et libre arbitre, in "Revue Philosophique de Louvain", 111, 1, 2013, pp. 27-52. Cfr. anche A. Gare, Approaches to the Question "What is Life?": Reconciling Theoretical Biology With Philosophical Biology, in "Cosmos and History", 4, 1-2, 2008, pp. 53-77.

${ }^{11}$ H. Jonas, Organismo e libertà, cit., p. 234. Sul concetto di transanimale, cfr. anche Id., Werkzeug, Bild und Grab. Vom Transanimalischen im Menschen, in Id., Philosophische Untersuchungen und metaphysische Vermutungen, Insel, Frankfurt am Main, 1992, e Suhrkamp, Frankfurt am Main, 1994, pp. 34-49. Nell'inedito citato alla nota precedente Jonas afferma che già per Aristotele la preminenza della vista risiede nel fatto che essa offre una "preferenza cognitiva" per ciò che permette di conoscere riguardo agli oggetti, sebbene non ancora riguardo al mondo dei significati (cfr. Nachlass, HJ-1-12-1, p. 21). Tra i sensi, Aristotele ne distinguerebbe pertanto alcuni (la vista e l'udito) in quanto “intrinsecamente più vicini a ciò che è specifico e distintivo dell'essere umano" (ivi, p. 22).
} 
fenomenologia dei sensi - della peculiarità della vista consente, ad avviso di Jonas, "di contribuire non solo alla fenomenologia dei sensi in se stessa, ma anche alla valutazione del loro ruolo nelle superiori prestazioni spirituali, che nel caso dell'uomo si basano su di essi” ${ }^{\prime 12}$. La tesi di fondo è infatti che le caratteristiche specifiche della vista (simultaneità rappresentativa, neutralizzazione della causalità dell'affezione sensoriale, distanza) ${ }^{13}$, benché necessitino di una base rigorosamente organica, riescano a sfuggire in qualche modo alla forza d'attrazione della materia, realizzino un grado più elevato di libertà del vivente ed esprimano quindi una forma più compiuta di dinamismo trascendente, culminante da ultimo nella spiritualità umana.

Non si può in questa sede ripercorrere dettagliatamente la fenomenologia dei sensi jonasiana che rende omaggio alla "nobiltà della vista"14. È però importante sottolineare quanto segue: il fatto che l'animale disponga del senso della vista evidenzia come la spinta al trascendimento radicata nelle viscere della vita assuma una forma specifica denominata da Jonas "transanimale"; e proprio il transanimale è ciò che può essere identificato come base materiale e sensitiva per le superiori attività spirituali umane ${ }^{15}$. Non che con ciò si pretenda di dedurre semplicemente e tout court queste ultime dalle caratteristiche possedute dall'animale ${ }^{16}$. Tuttavia, Jonas è

\footnotetext{
${ }^{12} \mathrm{H}$. Jonas, Organismo e libertà, cit., p. 180.

${ }^{13} \mathrm{Ibid}$.

${ }^{14}$ A proposito della vista, Jonas sottolinea anche come la nobiltà non debba degenerare in tirannia o nella pretesa di essere l'esclusiva depositaria di un certo valore: "Senza sudditi su cui regnare - afferma Jonas - un re non è più un re. La testimonianza della vista non falsa la realtà, se viene integrata dalla testimonianza degli altri strati dell'esperienza, soprattutto da quello della capacità motoria e del tatto; se essa rifiuta con arroganza il loro verdetto, allora la sua verità diviene sterile" (ivi, p. 194). A questo proposito Jonas ribadisce il giudizio di "parzialità della filosofia antica per uno dei sensi corporei" (ivi, p. 197). Ben al di là della questione concernente l'analisi della vista, Jonas si indirizza qui polemicamente contro un atteggiamento filosofico radicato in Occidente e tendente ad assolutizzare la centralità gnoseologica della visione. Jonas tratterà di questo tema anche nel saggio Visione e pensiero, in cui egli si misura con la percettologia di Rudolf Arnheim (cfr. H. Jonas, Philosophical Essays. From Ancient Creed to Technological Man, Prentice-Hall, Englewood Cliffs, 1974, poi University of Chicago Press, Chicago, 1980, tr. it. Dalla fede antica all'uomo tecnologico. Saggi filosofici, a cura di A. Dal Lago, Mulino, Bologna, 1991, pp. 327-342).

${ }^{15}$ Questo è, del resto, già anticipato in $\mathrm{H}$. Jonas, Organismo e libertà, cit., p. 180.

${ }^{16}$ In tal senso, il tentativo di individuare la base sensitiva di tali caratteri non si identifica affatto con la ricerca della loro origine materiale.
} 
fermamente convinto di come in questo dinamismo si possa individuare, per un verso, lo specifico dell'animale (e dunque la sua discontinuità rispetto ad altre forme viventi) e, per altro verso, anche la sua continuità nell'ambito del composito fenomeno della vita, continuità che giustifica l'interpretazione dell'inferiore (in questo caso, dell'animale) come prefigurazione del superiore (l'umano, lo spirituale, appunto). È significativo che da tutti e tre i summenzionati caratteri della vista (simultaneità, neutralizzazione e distanza) originino considerazioni di questo tipo e si aprano scenari "spirituali".

Jonas, infatti, nota innanzitutto come la facoltà immaginativa ${ }^{17}$ che si afferma pienamente con il senso della vista, ma che del resto è già prefigurata dal tatto, testimoni e, al tempo stesso, consenta un aumentato grado di autonomia dall'aderenza al dato materiale. In secondo luogo, la simultaneità percettiva della vista testimonia un fatto fenomenologico realmente gravido di possibilità "spirituali": dalla simultaneità è infatti concesso di muovere verso la temporalità, la quale - unitamente alla realtà del movimento - consente di distinguere e approdare a concetti quali l'essere e il divenire, per giungere da ultimo a concepire l'idea dell'eterno ${ }^{18}$. In terzo luogo, la tendenza della vista a neutralizzare la propria origine dinamica e causale consente di porre le premesse per la distinzione tra teoria e prassi, operazione che risulta impossibile agli altri sensi ${ }^{19}$. La distinzione desunta dalla percezione visiva aggiunge Jonas - tra "il concetto dell'oggettività della cosa come è in sé" e la "cosa

\footnotetext{
17 Cfr. H. Jonas, Organismo e libertà, cit., p. 186. Cfr. anche l'inedito jonasiano intitolato Life and Organism (Nachlass, HJ-1-12-1, p. 51). Come si vedrà, l'immaginazione si rivelerà centrale per l'antropologia jonasiana.

18 "Pertanto solo la vista fornisce la base sensitiva su cui lo spirito ha potuto per la prima volta concepire I'idea dell'eterno, di quello che non muta mai ed è sempre presente" (H. Jonas, Organismo e libertà, cit., p. 189).

19 "Nel tatto non abbiamo quindi quella chiara separazione fra la prestazione teorica dell'informazione e il comportamento pratico (liberamente basato su di essa) che abbiamo nella vista. Di nuovo scopriamo nella costituzione fondamentale di un senso e nelle sue condizioni fisiche la radice organica di una distinzione altamente spirituale a livello umano: la distinzione fra teoria e prassi" (ivi, p. 190).
} 
come mi influenza" è ciò da cui deriva "tutta l'idea di theoria e verità teoretica"20. Infine, la distanza spaziale rispetto a ciò che viene percepito, oltre ad aprire uno spazio di autonomia e libertà, mette il vivente dinanzi alla profondità dello spazio, che "è il luogo di nascita dell'idea dell'infinità, per la quale nessun altro senso ha saputo fornire la base empirica"21.

Lo studio della percezione animale risulta pertanto funzionale a mostrare come un'osservazione qualificata (vale a dire, condotta dal punto di vista umano, ovvero di un grado di vita "più sviluppato") del fenomeno animale-percettivo nel suo complesso consenta di individuarne l'essenza in una tendenza dell'interiorità emozionale a proiettarsi fuori di sé, oltreché in un rapporto di reciprocità (attività e passività) nei confronti della realtà. Ad avviso di Jonas, l'essere vivente

Riconosce perché qualcosa si offre al riconoscimento. L'azione ricettiva della percezione è accompagnata e resa possibile dall'azione spontanea dell'espressione. La seconda può anche costituire il fenomeno originale. La vita animale è espressiva, persino avida di espressione. Si mostra, ha i suoi segni codificati, il suo linguaggio; essa comunica se stessa ${ }^{22}$.

In virtù di tale osservazione qualificata l'essenza espressiva dell'animale assume il valore di testimonianza ontologica della propria potenzialità transanimale, la cui trattazione è al tempo stesso propedeutica per la comprensione dell'antropologico.

\section{Essere umano e animale}

20 Ivi, pp. 191-192. Poche pagine dopo, Jonas parla a questo proposito di "contemplazione 'disinteressata'” (ivi, p. 197). La neutralizzazione della propria origine dinamica da parte della vista viene da Jonas spiegata come segue: "Non ho bisogno di far altro che guardare - e l'oggetto non ne viene toccato; e non appena vi è luce l'oggetto ha solamente bisogno di esserci per essere anche visibile - e io non ne vengo toccato: e tuttavia esso viene percepito nel suo essere presso di sé a partire dal mio essere presso di me, esso mi è presente senza attirarmi nella sua presenza" (ivi, p. 191).

${ }^{21}$ Ivi, p. 196.

${ }^{22}$ H. Jonas, Dalla fede antica all'uomo tecnologico, cit., p. 353. Sui medesimi temi e con il medesimo intento di oltrepassare il dualismo tra esistenza umana e vita animale, cfr. anche F. Burgat, Liberté et inquiétude de la vie animale, Kimé, Paris, 2006; Ead., Une autre existence - La condition animale, Albin Michel, Paris, 2012. 
Come si è visto, l'analisi jonasiana culmina in un'articolata fenomenologia dei sensi. Questi ultimi, presi nel loro insieme presentano un carattere cinestetico, ma solo uno di essi, la vista, può vantare alcune proprietà esclusive. La sua nobiltà riassumibile nei tre attributi di simultaneità della presentazione, neutralizzazione dinamica della propria origine e superamento della distanza - non deriva solo dal suo essere la base materiale da cui nascono concetti filosofici fondamentali quali tempo, eternità, teoria e infinito, ma è pienamente giustificata dal fatto che attraverso la vista quel potenziale in cui consiste la libertà ontologica del vivente si realizza in maniera inedita nella forma dello spirito umano, il quale pertanto "è andato dove la vista ha indicato di andare" 23 .

Lo spirito umano avrebbe dunque in qualche modo portato a compimento le potenzialità transanimali tipiche dell'animale superiore. Questo solo fatto è di per sé sufficiente a rendere pienamente conto dell'essenza specifica dell'essere umano, e a provvederne una giustificazione in senso non-sostanzialistico, come auspicato da Jonas? A questo riguardo, l'orientamento di fondo della biologia filosofica jonasiana è di evitare il più possibile due estremi tendenti l'uno, per eccesso, a relegare tale essenza in una regione ontologica a sé, compiuta e conclusa (dualismo cartesiano), e I'altro, per difetto, a ridurre lo specifico qualitativo umano all'uniformità quantitativa e a-specifica - dell'essere cosmico (materialismo moderno) ${ }^{24}$. La sfida con cui Jonas si cimenta è pertanto duplice: essendosi innanzitutto premurato - come si è visto - di riattivare la relazione reale e costitutiva dell'essere umano con la propria base materiale e biologica, egli intende in secondo luogo procedere a una giustificazione ontologica della distinzione dell'uomo "dagli altri esseri viventi, quindi dall'animale" 25 .

\footnotetext{
${ }^{23}$ H. Jonas, Organismo e libertà, cit., p. 197.

${ }^{24} \mathrm{Cfr}$. ivi, p. 284. Su questi temi, mi permetto di rinviare a R. Franzini Tibaldeo, Limiti e legittimità della modernità in rapporto alla questione della libertà umana, in U. Perone (a cura di), Filosofia dell'avvenire, Rosenberg \& Sellier, Torino, 2010, pp. 28-36.

${ }^{25} \mathrm{H}$. Jonas, Organismo e libertà, cit., p. 204.
} 
Ebbene, a proposito dell'essere umano si avverte una complicazione: se, da un lato, la base del suo essere è indubbiamente biologica, dall'altro lato, la sua peculiarità ontologica risiede in un dinamismo centrifugo rispetto alla materia e al dato meramente biologico ${ }^{26}$. Questo fattore rende delicata la questione della scelta dei mezzi tramite cui individuare e riconoscere la specificità umana: occorre infatti sceglierne uno che sia in grado di indicare la differenza specifica dell'umano rispetto all'animale e, al tempo stesso, di valere come testimonianza dell'effettiva esistenza della libertà ontologica. Questa difficoltà - sembra dire Jonas - non emerge a proposito della specificità animale, rimanendo essa interamente nell'ambito del biologico, eccezion fatta per le "potenzialità transanimali", che tuttavia a questo livello rimangono, appunto, allo stato potenziale. L'esistenza dell'umano, al contrario, testimonia l'avvenuta realizzazione di tali potenzialità e, con ciò, anche il paradosso per cui, nell'ambito della dinamica biologica di autotrascendimento del dato materiale da parte della forma vivente, compare un nuovo livello ontologico che trascende tout court il biologico e non è riconducibile al precedente mediante una mera differenza di grado. Detta novità ontologica si manifesta comunque sempre a partire dal biologico, rimanendo in quest'ultimo costantemente radicato secondo una modalità che non cerca di nascondere la propria relazione a esso.

Come riconoscere dunque lo specifico dell'essere umano? A partire da quali caratteri? Per esemplificare meglio la questione, Jonas propone un esperimento mentale: alcuni astronauti, giunti su un pianeta sconosciuto, vogliono accertarsi se vi siano degli esseri umani. Stante quanto si è appena evidenziato, il mero riconoscimento biologico sarebbe insufficiente, poiché la peculiarità ontologica dell'essere umano consiste propriamente nella realizzazione di caratteri trans-materiali o trans-organici ${ }^{27}$. Pertanto, i mezzi di riconoscimento impiegati devono essere non

\footnotetext{
${ }^{26}$ Com'è noto, a questo proposito Helmuth Plessner parla di eccentricità (Exzentrizität) dell'essere umano.

27 “'Umano' - scrive qui Jonas - deve designare quindi qualcosa che giustifichi l'assegnazione del nome persino in presenza di un'estrema dissomiglianza fisica" (H. Jonas, Organismo e libertà, cit., p. 204).
} 
solo percettibili, convincenti, inequivocabili e primitivi, ma anche in grado di valere "indipendentemente dalla struttura organica" ${ }^{28}$. Poiché infine l'essere umano, rientrando nel novero degli esseri viventi, condivide con essi alcuni caratteri fondamentali della vita, tra cui quello di esprimere un rapporto attivo nei confronti del mondo, ecco che i mezzi di riconoscimento devono essere "un agire o il risultato di un agire"29, potendo in tal modo essere fenomenicamente osservabili e interpretabili come espressione di un'interiorità vivente.

A questo punto Jonas elenca varie manifestazioni tipicamente umane che potrebbero essere assunte a emblema dell'umanità dell'uomo: I'utensile, la tomba, il focolare, il linguaggio, la ragione, il pensiero, il raffigurare ${ }^{30}$. Il fatto che, in sintonia con i risultati della fenomenologia dei sensi, la scelta di Jonas ricada su quest'ultimo non significa che a esso si intenda conferire una qualche pretesa di primazia o esclusività testimoniale. Piuttosto, rispetto agli altri, esso presenta alcuni vantaggi ermeneutici, una relativa semplicità e una sicura riconoscibilità esteriore ${ }^{31}$.

Jonas impiega un metodo fenomenologico che, dallo studio dei caratteri di un'immagine, risale alle peculiarità dell'azione percettiva che ne costituiscono la ragion d'essere, per giungere infine a mettere in evidenza le caratteristiche di quel vivente che, in virtù dei requisiti in tal modo evidenziati, può essere indicato come produttore di immagini. I segni o le raffigurazioni di origine artificiale riscontrati infatti dagli esploratori sulle pareti di una caverna del pianeta sconosciuto valgono come indizi evidenti e probanti della "natura più-che-animale del suo artefice; e del fatto che

\footnotetext{
28 Ibid.

${ }^{29}$ lbid.

${ }^{30}$ Cfr. ivi, p. 205. Cfr. anche H. Jonas, Dalla fede antica all'uomo tecnologico, cit., pp. 361-362 e Id., Werkzeug, Bild und Grab, cit., pp. 37 ss.

${ }^{31}$ Altrove lo strumento sembra possedere una qualche priorità cronologica sull'immagine (cfr. H. Jonas, Dalla fede antica all'uomo tecnologico, cit., pp. 361-362).
} 
egli è un essere potenzialmente capace di parlare, pensare, inventare, in breve un essere 'simbolico'”'32.

La novità messa in campo dall'esistenza di immagini artificiali è infatti la loro inutilità biologica, non essendo la loro esistenza e la loro produzione dirette al perseguimento di alcuno scopo vitale ${ }^{33}$. Jonas sintetizza così una delle tesi centrali della sua antropologia: la rappresentazione figurativa costituisce un nuovo modo - non strettamente biologico - di appropriarsi dell'oggetto, ponendosi pertanto come un nuovo grado di mediatezza del rapporto tra essere vivente (in questo caso, l'uomo) e realtà ${ }^{34}$. A rendere possibile quest'inedita forma di relazione è il peculiare status dell'immagine artificiale, con la sua triplice stratificazione. Innanzitutto, l'immagine mostra di distinguersi dal proprio veicolo materiale (substrato) ${ }^{35}$. In secondo luogo sostiene Jonas - essa non coincide con l'oggetto raffigurato ${ }^{36}$. Ne consegue che l'immagine acquisisce la possibilità di venire considerata per sé e come

\footnotetext{
${ }^{32}$ H. Jonas, Organismo e libertà, cit., pp. 205-206. Già in Augustin und das paulinische Freiheitsproblem (1930) Jonas identifica nella "simbolicità" la struttura fondamentale dello spirito (cfr. H. Jonas, Augustin und das paulinische Freiheitsproblem. Ein philosophischer Beitrag zur Genesis der christlichabendländischen Freiheitsidee, Vandenhoeck \& Ruprecht, Göttingen, 1930, seconda edizione riveduta e ampliata, con il titolo Augustin und das paulinische Freiheitsproblem. Eine philosophische Studie zum pelagianischen Streit, con un'introduzione di J. M. Robinson, Vandenhoeck \& Ruprecht, Göttingen, 1965, tr. it. Agostino e il problema paolino della libertà. Studio filosofico sulla disputa pelagiana, a cura di C. Bonaldi, Morcelliana, Brescia, 2007, pp. 113-114). Su questo aspetto, cfr. D. Böhler, Hans Jonas Stationen, Einsichten und Herausforderungen eines Denklebens, in Id. (a cura di), Ethik für die Zukunft: im Diskurs mit Hans Jonas, München, Beck, 1994, pp. 45-67, qui pp. $52-53$ e F. Bianco, Hans Jonas tra Heidegger e Bultmann, in "Paradigmi", 22, 66, 2004, pp. 303-317, qui pp. 307-308.

${ }^{33}$ Tra gli scopi vitali utili, Jonas cita l'alimentazione, la riproduzione, il nascondersi e lo svernare (cfr. $\mathrm{H}$. Jonas, Organismo e libertà, cit., p. 206). Egli poi continua come segue: "La rappresentazione di qualcosa non trasforma né l'ambiente, né la condizione dell'organismo stesso. Un essere che crea immagini indulge pertanto alla produzione di cose inutili o ha degli scopi al di là di quello biologico o può perseguire questi ultimi anche in un modo diverso che non attraverso l'impiego strumentale di cose" (ibid.).

${ }^{34}$ Questo grado di mediatezza (mediacy, Mittelbarkeit) è il terzo, dopo quello metabolico e quello percettivo-attivo-animale (cfr. ivi, p. 235). La scelta jonasiana di eleggere a cifra dell'umanità dell'uomo un aspetto biologicamente "inutile" vale dunque come pendant del suo rifiuto di ritenere - come fa invece l'evoluzionismo darwiniano - che l'essenza del fenomeno della vita consista nella mera sopravvivenza.

${ }^{35}$ Cfr. ivi, pp. 211-212.

${ }^{36} \mathrm{Cfr}$. ivi, p. 212.
} 
ontologicamente indipendentemente da qualsiasi commistione materiale ${ }^{37}$. Jonas pertanto conclude che essa si libra fra le altre due entità reali "come una terza entità ideale" 38 o spirituale.

A questo punto Jonas si domanda quali siano le proprietà richieste in un soggetto per produrre e cogliere immagini. L'obiettivo è di esplicitare - a partire dal fenomeno della rappresentazione figurativa - in che cosa consista la facoltà rappresentativa umana, che può valere come indizio della specificità umana.

Due sono i risultati messi a segno da Jonas. In primo luogo, ottiene la conferma del fatto che la potenzialità transanimale rivelatasi nel senso della vista abbia trovato specifica attuazione nell'atto raffigurativo intenzionale (con la sua capacità di separare la forma raffigurata dalla materia) ${ }^{39}$. In secondo luogo, a partire dallo studio delle condizioni di possibilità del percepire e del produrre immagini, è possibile risalire alla facoltà del percepire la somiglianza, la quale, a sua volta, è in grado di indicare il proprio oltre, vale a dire la "facoltà più fondamentale di separare l'eidos dall'esistenza, o la forma dalla materia" ${ }^{40}$. È pertanto proprio dal moto astraente insito nella percezione - spiega Jonas - che nasce l'immagine come forma (eidos) "di ordine superiore" ${ }^{41}$, che si mantiene a distanza rispetto allo stesso atto percettivo, conservandone simultaneamente l'unità e l'identità. In tal senso, l'immagine eidetica e intenzionale è in grado di valere anche come simbolo dell'oggetto. Jonas può così concludere che "astrazione, rappresentazione, simbolismo

\footnotetext{
${ }^{37}$ A questo proposito Jonas parla di "differenza ontologica" (ibid.).

${ }^{38} \mathrm{Ibid}$. Poche pagine dopo Jonas ribadisce infatti come in origine "eidos" significasse solo "apparenza" o "aspetto" (ivi, p. 215), acquisendo solo successivamente il significato di rappresentazione, idea o forma.

39 La separazione intenzionale di forma e materia è, infatti, ciò che "rende possibile la presenza figurativa del fisicamente assente insieme all'autonegazione del fisicamente presente" (ivi, p. 214).

${ }^{40}$ IVi, p. 215.

${ }^{41}$ Ivi, p. 217. La superiorità dell'eidos viene successivamente riassunta nella sua universalità, vale a dire nella sua indipendenza simbolica da un qui e un ora specifici. Divenendo universale, l'immagine (e poi, successivamente, il nome) è in grado di replicare ogni volta quella prima azione umana simbolica ricreativa del mondo, cui fa riferimento anche la Bibbia (cfr. Genesi, 2,19), la quale tuttavia, limitando - ad avviso di Jonas - la propria considerazione ai nomi, non coglie il loro presupposto indiscutibile, cioè "la disponibilità dell'eidos al di là delle cose singole per la comprensione, l'immaginazione e il discorso umani" (H. Jonas, Organismo e libertà, cit., p. 221).
} 
- qualcosa della funzione dell'immagine - sono inerenti alla vista, in quanto essa è il più integrativo dei sensi. In certo grado ciò deve venire riconosciuto già agli animali superiori" ${ }^{42}$.

Jonas ha così posto le premesse per affrontare il nucleo centrale della propria analisi, vale a dire l'individuazione della specificità umana, che mostra un duplice volto. Posto infatti che la percezione transanimale pienamente dispiegata e sviluppata ha la capacità di cogliere l'apparenza come apparenza, cioè come distinta dalla realtà e disponendo liberamente della sua presenza, ne segue un duplice risultato. Per un verso, infatti, tale libera disposizione si realizza "nell'esercizio interiore della facoltà immaginativa" ${ }^{43}$, in virtù di cui il ricordo umano si distingue effettivamente dalla memoria animale per la libertà di riflettere sulle cose nell'immaginazione, vale a dire per una libertà della distanza e del dominio rispetto a ciò che viene meramente percepito ${ }^{44}$. Per altro verso, però, la forma ricordata e immaginata viene trasposta anche in immagine esteriore e materiale, la quale può a sua volta divenire oggetto di percezione anche da parte di altri esseri umani. Il dinamismo che così si instaura tra interiorità ed esteriorità non ricade però in una forma dualistica, dal momento che tali dimensioni costituiscono i poli entro i quali ha luogo una dinamica unitaria ${ }^{45}$.

A proposito della libertà umana e del suo carattere unitario, benché polare, ci si trova in presenza di un'analoga polisemia. Essa si manifesta come decisione, cioè come spinta a trascendere la realtà attuale verso un "regno del possibile, che può venire realizzato dall'uomo a sua scelta" ${ }^{\prime 46}$. Inoltre, in quanto simbolica, la libertà si manifesta espressamente nell'attività figurativa e nella produzione di immagini.

\footnotetext{
${ }^{42}$ Ivi, p. 217.

${ }^{43}$ Ivi, p. 218.

${ }^{44}$ La memoria animale è infatti sempre legata alla sensazione attuale e "nulla depone a favore del fatto che questo tipo di memoria abbia immaginativamente presenti i suoi oggetti e tutto depone contro l'ipotesi che - anche se così fosse - questa presenza starebbe a disposizione del soggetto per venire citata e abbandonata a piacimento" (ibid.). Al contrario, il "ricordo umano trascende tale memoria attraverso la libera facoltà riproduttiva dell'immaginazione, che domina le immagini delle cose" (ibid.). ${ }^{45}$ In questo modo si giustifica tra l'altro la possibilità di risalire dall'esteriorità fenomenica all'interiorità. ${ }^{46} / v i$, p. 220.
} 
La produzione esteriore di immagini interiori testimonia dunque - ad avviso di Jonas - un tratto specificamente umano, vale a dire il tipo di controllo che l'uomo ha del suo corpo, che, da ultimo, è riconducibile alla medesima modalità con cui l'uomo produce liberamente le immagini interiori stesse. La libertà motoria attivata a proposito della produzione di immagini replica quella immaginativa: "il controllo interiore dell'eidos" ha "anche il potere di guidare il corpo nel corso dell'esecuzione. Solo così l'immaginazione [Vor-stellung] può progredire a rappresentazione [Darstellung]" 47 . Come esempi di tale "trasposizione" dell'interiore in movimenti corporei, Jonas cita lo scrivere, la danza e l'uso della mano, che sta all'origine della tecnica. Avendo in tal modo proceduto a raccordare interiorità ed esteriorità, sottolineandone la rilevanza ai fini della chiarificazione del ruolo della libertà, Jonas suggella il proprio ragionamento con queste righe particolarmente efficaci:

Quello che abbiamo qui dinnanzi è un dato di fatto transanimale, peculiarmente umano: il controllo eidetico della motilità, cioè l'attività muscolare comandata non da schemi fissi di stimolo-risposta, bensì da una forma liberamente scelta, interiormente immaginata e intenzionalmente proiettata. Il controllo eidetico della motilità, con la sua libertà di esecuzione esterna, integra così il controllo eidetico dell'immaginazione, con la sua libertà di progettazione interna. Senza quest'ultima non vi sarebbe facoltà razionale, ma senza la prima il suo possesso sarebbe inutile, perché privo d'effetto. Tutte e due insieme rendono possibile la libertà dell'uomo ${ }^{48}$.

II fatto che - per Jonas - l'evidenza della libertà umana sia testimoniata dalla semplice esistenza delle immagini ${ }^{49}$, non deve, tuttavia, essere inteso come un indebolimento della differenza ontologica dell'umano rispetto agli altri viventi e all'animale. A scongiurare infatti tale eventualità, Jonas si affretta a rimarcare che "lo

\footnotetext{
47 Ibid. Questo significativo gioco di parole non compare nell'edizione in lingua inglese di The Phenomenon of Life (1966).

${ }^{48}$ H. Jonas, Organismo e libertà, cit., p. 220. Cfr. a questo proposito anche Id., Werkzeug, Bild und Grab, cit., pp. 38-39 e 43-44.

49 Jonas scrive infatti che l'“esistenza delle immagini, la quale mostra la forma sottratta al fatto, testimonia questo livello [di una mediatezza non animale; N.d.R.] e nella sua illimitata promessa è sufficiente come evidenza della libertà umana" (H. Jonas, Organismo e libertà, cit., p. 222).
} 
iato fra rapporto animale verso il mondo e il più primitivo tentativo di una rappresentazione è infinitamente più grande di quello fra quest'ultimo e ogni costruzione geometrica. È uno iato metafisico, a paragone del quale l'altro è solo una differenza di grado 50 .

D'altro canto però si è anche visto come lo studio del transanimale, che pure apre all'autentica e reale distinzione dell'umano rispetto all'animale, non debba consumarsi in una separazione dualistica dell'essere umano dal contesto naturale e complessivo del vivente ${ }^{51}$. Come già anticipato, l'obiettivo di Jonas è di tutelare l'essenza dell'uomo caratterizzandola come una differenza qualitativa la cui comparsa nella storia evolutiva sia imprevedibile e non conseguibile per via di mera deduzione dagli stadi viventi "inferiori", e mostrandone al tempo stesso il radicamento biologico e la solidarietà ontologica con il vivente. Entrambi i lati della questione sono garantiti dalla complessiva e articolata rivoluzione ontologica operata dall'autore, che recupera - in una veste profondamente rinnovata - concetti aristotelici quali potenza (potenzialità) e atto (effettiva realizzazione, forma) ${ }^{52}$.

In ogni caso, il fatto che a proposito della distinzione tra essere umano e animale l'autore richiami un'implicazione di carattere metafisico significa che per certi aspetti il pensiero jonasiano resta inequivocabilmente segnato da una forma di antropocentrismo, di cui non si può in questa sede analizzare esaustivamente le ragioni

\footnotetext{
${ }^{50} \mathrm{Ivi}$, p. 223. Jonas ribadisce i medesimi concetti anche in Werkzeug, Bild und Grab, cit., p. 36. Cfr. anche H. Jonas, Dalla fede antica all'uomo tecnologico, cit., p. 337. Secondo l'autore, la dicotomia tra animale e uomo, assumendo rilevanza metafisica, risulta assai più profonda e significativa rispetto alle precedenti discontinuità riscontrabili nel fenomeno della vita (cfr. H. Jonas, Organismo e libertà, cit., p. 223). Sotto questo aspetto, Jonas mostra di essere in sintonia con la tradizione ebraico-cristiana, secondo cui l'essere umano e il resto della natura si distinguono per il fatto che il primo è "creato a immagine di Dio, mentre il resto della creazione [animali compresi; N.d.R.] è puramente l'opera di Dio". Dunque i cieli "non sono la gloria, ma la narrano" (cfr. H. Jonas, Problemi di libertà, a cura di E. Spinelli, con la collaborazione di A. Michelis, Aragno, Torino, 2010, p. 110).

${ }^{51}$ Sotto questo riguardo, si può - tra l'altro - notare la vicinanza di Jonas all'antropologia filosofica novecentesca (in particolare alle riflessioni di Gehlen e Plessner), oltreché alle ricerche biologiche condotte da Adolf Portmann (cfr. in particolare A. Portmann, Aufbruch der Lebensforschung, Rhein, Zürich, 1965, tr. it. Le forme viventi, Adelphi, Milano, 1969). Cfr. anche F. Burgat, Liberté et inquiétude de la vie animale, cit.; J. Fischer, Philosophische Anthropologie, cit.; F. Burgat, Une autre existence, cit.

${ }^{52}$ Su questo aspetto, mi permetto di rinviare al mio La rivoluzione ontologica di Hans Jonas, cit.
} 
e le conseguenze etiche ${ }^{53}$. È però evidente che uno degli elementi che meglio evidenzia il senso e la rilevanza di tale iato metafisico sia l'esperienza - tipicamente ed esclusivamente umana, appunto - della responsabilità: I'essere umano è infatti il solo a essere responsabile e, in senso stretto, imputabile per le sue azioni (individuali o collettive), i cui effetti potenzialmente devastanti gettano un'ombra sull'avventura ontologica della vita in quanto tale ${ }^{54}$.

\section{La posizione dell'animale nel cosmo}

Chiarito dunque il rapporto che - ad avviso di Jonas - lega l'essere umano all'animale e avendo ripetutamente accennato al fatto che il cosiddetto "fenomeno della vita" presenterebbe un senso ontologico complessivo, si può dar seguito alle precedenti riflessioni chiedendosi: in che cosa consiste precisamente tale senso ontologico complessivo? Quale forma assume? E poi: quale ruolo gioca, ad avviso di Jonas, il mondo vivente non umano (e in specie quello animale) in quel disegno ontologico complessivo? In altre parole, quale posizione occupa il vivente animale nell'avventura cosmica della vita?

\footnotetext{
${ }^{53}$ Sulla problematica dell'antropocentrismo, anche in riferimento all'etica jonasiana, cfr. C. Foppa, L'être humain dans la philosophie de la biologie de Hans Jonas: quelques aspects, in G. Hottois-M.-G. Pinsart (a cura di), Hans Jonas. Nature et responsabilité, Vrin, Paris, 1993, pp. 169-189, tr. it. L'essere umano nella filosofia della biologia di $\mathrm{H}$. Jonas: qualche aspetto, in Hans Jonas. Natura e responsabilità, a cura di P. Pellegrino, Milella, Lecce, 1995, pp. 245-267; P. Destrée, Finalité et subjectivité. Quelques remarques sur l'anthropomorphisme, in "Études phénoménologiques", 23-24, 1996, pp. 209-224; W. Szostak, Teleologie des Lebendigen: zu K. Poppers und H. Jonas' Philosophie des Geistes, Lang, Frankfurt am Main, 1997, pp. 200 ss.; A. Pessina, Bioetica e ontologia. Nota sul pensiero di Hans Jonas, in "Ragion pratica", 8, 15, 2000, pp. 131-148, qui p. 146; V. Hösle, Ontology and Ethics in Hans Jonas, in "Graduate Faculty Philosophy Journal", 23, 1, 2001, pp. 31-50, qui pp. 37-38; J. Dewitte, L'anthropomorphisme, voie d'accès privilégiée au vivant. L'apport de Hans Jonas, in "Revue Philosophique de Louvain", 100, 3, 2002, pp. 437-465; P. Piccolella, II limite di Prometeo: pensare uomo, natura e Dio con Hans Jonas, Lithos, Roma, 2006, pp. 158-163; G. De Marzo, Processi organici e razionalità. Il sistema jonasiano, Pensa Multimedia, Lecce, 2008; M. Reichlin, Etica della vita. Nuovi paradigmi morali, Bruno Mondadori, Milano, 2008, p. 176; R. Franzini Tibaldeo, La rivoluzione ontologica di Hans Jonas, cit., pp. 323-325.

54 Cfr. H. Jonas, Organismo e libertà, cit., 285-304; Id., II principio responsabilità, cit. Per un'interpretazione della "transanimalità" dell'uomo come premessa al principio responsabilità, cfr. J. Oliveira, A "transanimalidade" do homem: uma premissa do princípio responsabilidade, in R. dos SantosJ. Oliveira-L. Zancanaro (a cura di), Ética para a civilação tecnológica: em diálogo com Hans Jonas, Centro Universitário São Camilo, São Paulo, 2011, pp. 41-60.
} 
II mutamento di prospettiva impresso da questi interrogativi è legato al fatto che, da sola, la biologia filosofica non è in grado di affrontarli. Come già evidenziato, però, la biologia filosofica non può essere semplicemente accantonata, e ciò per il semplice fatto che proprio a partire dai suoi risultati conoscitivi è stato possibile leggere nel fenomeno della vita un moto ontologico di autotrascendimento che nella sua forma più articolata e complessa - l'essere umano - tende verso una dimensione metafisica, già peraltro ravvisabile anche quale principio e sorgente di senso dello stesso fenomeno della vita ${ }^{55}$. Piuttosto che un abbandono della biologia filosofica, ciò che si richiede sembra piuttosto un suo completamento ipotetico e per via suppositiva (ma pur sempre verosimile; un'integrazione peraltro postulata dall'esigenza di completezza esplicativa del fenomeno della vita), non essendo qui disponibile alcun'altra forma di conoscenza ${ }^{56}$. Per questa delicata operazione Jonas attinge al

\footnotetext{
${ }^{55}$ Con le parole di Jonas: "Il mistero stesso del divenire non ci è accessibile: resta perciò una congettura - per me personalmente una solida ipotesi -, che già il principio che fonda il passaggio dalla sostanza inanimata a quella vivente sia una tendenza caratterizzabile in questo senso nelle profondità dell'essere stesso. Ma il ricorso a tale concetto si mostra però immediatamente adeguato per la descrizione della struttura vivente più elementare" (H. Jonas, Organismo e libertà, cit., p. 116; cfr. anche Id., Erinnerungen. Nach Gesprächen mit Rachel Salamander, a cura di Ch. Wiese, Insel, Frankfurt am MainLeipzig, 2003, tr. it. Memorie. Conversazioni con Rachel Salamander, Melangolo, Genova, 2008, p. 291). E il Nostro continua in nota: "La deduzione dalla validità descrittiva di quella eziologica è un ardimento della speculazione. Veniamo spinti a ciò quando teniamo già conto - come dobbiamo -, nel suo primo non ancora sviluppato manifestarsi, della travolgente logicità con cui il principio si evolve in avanti con figure sempre più ardite che lo rappresentano in modo sempre più completo e quando cogliamo con ciò a ritroso anche i suoi antecedenti, che devono sostenere il tutto: la produttiva e visibile tendenza allo scopo del processo di ampia portata rende altamente improbabile una pura 'eterogonia dei fini' in relazione al suo inizio. Così con il concetto ontologico di libertà ci ritroviamo a dipendere dalla materia, nella quale gli scopi non sono visibili, la quale tradisce però la sua segreta potenzialità nella temeraria avventura della vita. Così come secondo la testimonianza della vita la sua fissa e non libera identità di sé non è l'ultima parola dell'essere, tanto meno ha bisogno di esserne la prima. Una storia metafisica della 'sostanza' potrebbe trascenderla da entrambi i lati... Così veniamo inevitabilmente costretti a un'interpretazione speculativa dell'essere in generale, in cui la materia trova il suo posto come modo o stato dell'essere stesso, come fase ontologica. Per il momento abbiamo a che fare con un'interpretazione rigorosamente fenomenologica della vita" (H. Jonas, Organismo e libertà, cit., pp. 116-117). Cfr. anche ivi, pp. 285 ss. e H. Jonas, Materie, Geist und Schöpfung. Kosmologischer Befund und kosmogonische Vermutung, Suhrkamp, Frankfurt am Main, 1988, tr. it. Materia, spirito e creazione. Reperto cosmologico e supposizione cosmogonica, a cura di P. Becchi e R. Franzini Tibaldeo, Morcelliana, Brescia, 2012.

56 Sullo statuto epistemologico di tale conoscenza, che per Jonas assume il volto del "mito", cfr. ivi, p. 301 e H. Jonas, Heidegger and Theology, in "The Review of Metaphysics", 18, 1964, pp. 207-233,
} 
proprio retroterra culturale ebraico, oltreché alle sue reminiscenze di studioso delle religioni antiche, ed elabora un "mito ipotetico" circa le origini dell'universo ${ }^{57}$. L'aspetto rilevante di questo mito è che la creazione divina sarebbe avvenuta per autonegazione e autoalienazione da parte della divinità stessa ${ }^{58}$. E ciò al fine di consentire che la vita si dispiegasse in tutta la sua ambivalente varietà e libertà, fino a giungere alla sua manifestazione tipicamente umana ${ }^{59}$, cioè quella contrassegnata dalla duplice possibilità di salvare o rovinare ciò che essa fa di sé, del mondo e dell'avventura divina stessa.

Eppure, secondo il mito jonasiano, l'esistenza del mondo e la comparsa della vita non sono accadimenti che seguono necessariamente l'auto-negarsi di Dio e l'affidarsi "al caso, al rischio e all'infinita varietà del divenire"60. Jonas infatti ribadisce che "non vi è alcuna necessità che in generale un mondo esista" 61 , né - potremmo aggiungere - che il cosmo debba presentare tali e tante forme viventi, ivi comprese quelle animali e quella umana. Conseguentemente, "Nessun dovere incondizionato

versione tedesca Heidegger und die Theologie, in "Evangelische Theologie", 24/12, 1964, pp. 621-642, tr. it. Heidegger e la teologia, a cura di R. Franzini Tibaldeo, Medusa, Milano, 2004, pp. 74-77.

57 Jonas presenta il proprio "mito ipotetico" nel saggio Immortalità ed esistenza odierna (cfr. H. Jonas, Organismo e libertà, cit., pp. 285-304) e in Id., Der Gottesbegriff nach Auschwitz. Eine jüdische Stimme, Suhrkamp, Frankfurt am Main, 1987, versione originaria in lingua inglese in A. H. Friedlander (a cura di), Out of the Whirlwind, Union of American Hebrew Congregations, New York, 1968, pp. 465-476, tr. it. II concetto di Dio dopo Auschwitz. Una voce ebraica, a cura di C. Angelino, Melangolo, Genova, 1989, 19932 ${ }^{2}$, pp. 23-27. Per le ascendenze qabbalistiche del mito jonasiano, cfr. ivi, pp. 37 e 42 . Sul mito ipotetico jonasiano, cfr. tra gli altri N. Russo, La biologia filosofica di Hans Jonas, Guida, Napoli, 2004, pp. 153-179; C. Bonaldi, Hans Jonas e il mito. Tra orizzonte trascendentale di senso e apertura alla trascendenza, Mercurio, Vercelli, 2007.

${ }^{58} \mathrm{Si}$ vedano le acute osservazioni di Nathalie Frogneux al riguardo (N. Frogneux, Hans Jonas ou la vie dans le monde, De Boeck \& Larcier, Bruxelles, 2001, pp. 257-260).

59 Nelle parole di Jonas: "L'avvento dell'uomo significa l'avvento del sapere e della libertà e con questa dote estremamente a doppio taglio, l'innocenza del mero soggetto della vita che realizza se stessa fa posto al compito della responsabilità disgiungendo bene e male" (H. Jonas, Organismo e libertà, cit., p. 300). Cfr. Id., Problemi di libertà, cit., p. 114. Cfr. anche N. Frogneux, Une liberté responsable et décentrée à l'égard de la nature. Lecture anthropologique du "Principe responsabilité", in "L'art du comprendre", Vol. La nature et son souci, no. 21, 2012, pp. 165-185; R. Franzini Tibaldeo, From Dualism to the Preservation of Ambivalence. Hans Jonas' "Ontological Revolution" as the Background to his Ethics of Responsibility, in C. Larrère-E. Pommier (a cura di), L'éthique de la vie chez Hans Jonas, Publications de la Sorbonne, Paris, 2013, pp. 33-48.

${ }^{60}$ H. Jonas, Organismo e libertà, cit., p. 298.

61 Ivi, p. 302. 
che l'uomo debba esistere è fondabile in base al caso cosmico del divenire, per cui egli esiste" ${ }^{\prime 2}$. Eppure la sola esistenza di fatto di tutta la ricchezza di manifestazioni viventi pre-umane e umane, benché conservi un aspetto inevitabilmente contingente (avrebbe potuto non essere o avrebbe potuto essere diversamente), esprime un valore, il cui significato vale come testimonianza di un senso eccedente ontologico e da ultimo metafisico ${ }^{63}$. In virtù di ciò, la sola comparsa, nel regno della materia, del fenomeno della vita unitamente alla sua ricchezza morfologica fa di esso un evento di portata qualitativamente superiore e attraverso cui traluce una questione eterna.

Detto altrimenti, nella dinamica processuale e cosmica è - ad avviso di Jonas possibile ravvisare una "rivoluzione ontologica" 64 , che coincide con la comparsa del vivente. L'esistenza di quest'ultimo è dunque contrassegnata da un intrinseco valore ontologico, in virtù di cui ogni vivente può reclamare, dinanzi a quel particolare vivente che è l'uomo, di essere rispettato e di diventare oggetto della sua responsabilità. Questo richiamo etico proveniente dalla "totalità minacciata del mondo vivente" prende la forma di un "muto appello"65 a preservare l'integrità della natura, dalla cui ricchezza e dal cui sviluppo lo spirito umano (l'unico in grado di rispondere a tale appello) ${ }^{66}$ è sorto e si è manifestato.

Che ruolo ha dunque l'animale in tutto ciò? In quanto titolare di "potenzialità transanimali" suscettibili di ulteriore sviluppo, la forma vivente animale è ciò senza di cui l'essere umano non sarebbe potuto esistere. La comparsa di quest'ultimo rappresenta poi - ipotizza il mito jonasiano - il momento in cui la trascendenza e la sua scelta di affidarsi al "gioco d'azzardo dell'evoluzione" si risvegliano a se stesse e accompagnano l'agire umano "con il fiato sospeso, sperando e aspirando, con gioia e

\footnotetext{
62 Ivi, p. 303.

${ }^{63} \mathrm{Cfr}$. a questo riguardo H. Jonas, Materia, spirito e creazione, cit., §§ 4-5.

${ }^{64} \mathrm{Cfr}$. H. Jonas, Memorie, cit., p. 285 e Id., Organismo e libertà, cit., p. 114.

${ }^{65} \mathrm{H}$. Jonas, Il principio responsabilità, cit., p. 12.

66 Non a caso, anche da un punto di vista etimologico il concetto di "responsabilità" (dal latino "respondĕo"; in tedesco "Verantwortung") si presenta costruito intorno al proprio nucleo "responsivo" (l'italiano "risposta" corrisponde al tedesco "Antwort"). Cfr. R. Franzini Tibaldeo, Responsabilità, in "Lessico di etica pubblica", 3, 1, 2012, pp. 183-200.
} 
dolore, con soddisfazione e delusione e, come suppongo, rendendosi a esso percepibile"67. Benché con la comparsa dell'essere umano cresca anche il pericolo, è comunque indubbio che "la trascendenza cresce con il raccolto terribilmente ambiguo delle nostre azioni”68.

Questa appare dunque come la cifra conclusiva del ragionamento jonasiano: non l'omega di un'apocatastasi definitiva o di una restaurazione finale, quanto piuttosto uno stare con il fiato sospeso nello spazio ambivalente della libertà. All'inizio dei tempi, la creazione per autonegazione e autoalienazione ha lasciato spazio al divenire e ai ghirigori della vita. Nulla si dice però della fine ultima o del fine ultimo, se non dal punto di vista di un pensiero, che sa di essere perennemente in cammino, e nella forma di un sentimento ambivalente, misto di paura e speranza: paura, dinanzi alla possibilità che l'essere umano, macchiandosi di un delitto ontologico, si renda responsabile del fallimento dell'impresa divina e dell'annichilimento della vita cosmica; speranza, dinanzi alla possibilità che egli risponda alle attese della divinità e si attivi per preservare la dignità, l'integrità e il pluriverso del cosmo e delle sue forme viventi.

La scelta jonasiana di sostare speculativamente entro l'ambiguità itinerante della libertà ontologica è all'origine del suo disinteresse per questioni, quali l'apocatastasi finale e la "sopravvivenza della persona in un aldilà futuro" ${ }^{69}$, dottrina che viene da Jonas criticata ${ }^{70}$. E neppure per gli animali si configura un ruolo specifico nell'eternità. Essi hanno però un duplice ineliminabile valore: per un verso, contribuiscono alla ricchezza e alla pienezza del cosmo; per altro verso, con la loro dote di potenzialità preannunciano l'umano e ne accompagnano il cammino ${ }^{71}$.

\footnotetext{
${ }^{67}$ H. Jonas, Organismo e libertà, cit., p. 300.

68 Ivi, p. 301.

${ }^{69}$ Ivi, p. 288.

${ }^{70}$ Cfr. ivi, pp. 288-290. Cfr. anche H. Jonas, Materia, spirito e creazione, cit., § 3. Sul medesimo tema, cfr. L. Vogel, Hans Jonas's Exodus: From German Existentialism to Post-Holocaust Theology, Introduction, in $\mathrm{H}$. Jonas, Mortality and Morality. A Search for the Good after Auschwitz, Northwestern University Press, Evanston, 1996, pp. 3-40, qui pp. 22-24; N. Frogneux, Hans Jonas ou la vie dans le monde, cit., p. 248.

${ }^{71} \mathrm{Cfr}$. E. Spinelli, Hans Jonas e la responsabilità verso il mondo animale, in "La rassegna mensile di Israel", 88, 1-2, 2012, pp. 31-46.
} 
All'essere umano è toccato in sorte di collaborare con la divinità, affinché essa riacquisti la propria pienezza e possa così giungere a riscattarsi completamente. Sotto questo riguardo, è essenziale che la responsabilità umana si adoperi non in vista dell'apocatastasi finale, ma per il mantenimento della possibilità di futuro.

Doutor em Filosofia Università degli Studi di Torino - Dipartimento di Filosofia e Scienze dell'Educazione Scuola Superiore Sant'Anna, Pisa E-mail: fratiba@yahoo.com 